\title{
Unusual Growth of InP Nanowires Grown on Silicon Surfaces
}

\author{
Ibrahim Kimukin, Chad D. Johns, Christopher \\ W. Edgar and M. Saif Islam \\ Department of Electrical and Computer Engineering, \\ University of California, Davis, CA, 95616, USA \\ Email: saif@ece.ucdavis.edu
}

\author{
Sungsoo Yi \\ Molecular Technology Laboratories, Agilent \\ Technologies, Palo Alto, CA 94304, USA \\ Currently address: Advanced Laboratories, Lumileds \\ Lighting, San Jose, CA 95131, USA
}

\begin{abstract}
Heteroepitaxial growth of III-V compound semiconductors on Si would enable the integration of high speed optoelectronic devices with mature Si technology. We report the growth of single-crystalline InP nanowires on (111)-oriented $\mathrm{Si}$ surfaces by chemical vapor deposition catalyzed by Au particles. Unusually fast growth of both catalyzed and un-catalyzed InP nanowires was observed. Understanding the unusual growth of these nanowires would enable us to solve the issues faced in the growth of III-V compound semiconductor on a Si surface.
\end{abstract}

Keywords: Nanowire, InP, Si, MOCVD Growth.

\section{INTRODUCTION}

Integration of high performance III-V compound semiconductors into mature Si technology is the focus of many intense researches. However, the growth of thin layers of these compound semiconductors on $\mathrm{Si}$ is still challenging due to several difficulties such as lattice mismatch, difference in crystal structure, and different thermal expansion coefficient $[1$, 2]. On the other hand, vapor-liquid-solid (VLS) growth method has opened opportunities for growing nanowire (NW) heterostructures with large lattice mismatch such as InAs on InP (3.1\% lattice mismatch) [3], GaAs on GaP (3.1\% lattice mismatch) [4], and $\mathrm{InP}$ on $\mathrm{Si}$ (8.0\% lattice mismatch) [5]. These results show that lattice mismatch can be accommodated in the nanowire structures because of their small cross-section and, thus, address the long-standing challenge of integrating high performance III-V semiconductors with mainstream Si technology.

In this work, we report the epitaxial growth of Au-catalyzed and catalyst-free InP nanowires on $\mathrm{Si}$. We show that the density and the size of the catalyst determine the growth rate and the size of the nanowires. Additionally, unusually fast growth of catalyst free InP nanowires on $\mathrm{Si}$ is reported for the first time.

\section{GROWTH}

We used (111)-oriented Silicon wafers for the vertical growth of the nanowires. Initially samples were cleaned with hydrofluoric acid (HF). Then some areas were defined on the sample with photolithography. Finally the samples were deposited with nominally $0.5 \mathrm{~nm}$ (sample 1 ) and $1 \mathrm{~nm}$ (sample 2) thick gold layer using electron-beam evaporation technique on the defined area.
The InP NWs were grown in a vertical MOCVD reactor equipped with a close-coupled showerhead. The reactor was typically operated at 76 Torr with a total $\mathrm{H}_{2}$ flow of $10 \mathrm{slm}$. Before the growth samples were annealed at $650{ }^{\circ} \mathrm{C}$ in $\mathrm{H}_{2}$ for $10 \mathrm{~min}$. to form $\mathrm{Au}$ catalyst nanoparticles from the deposited $\mathrm{Au}$ film. The reactor temperature was then lowered to the typical growth temperature of $450{ }^{\circ} \mathrm{C}$, and trimethylindium $\left(\operatorname{In}\left(\mathrm{CH}_{3}\right)_{3}\right)$ and phosphine $\left(\mathrm{PH}_{3}\right)$ were introduced into the reactor to initiate the growth of InP nanowires. The growth duration was 10 min and a typical growth rate was found to be $\sim 0.1 \mu \mathrm{m} / \mathrm{min}$. transmission electron microscope (TEM) investigations found the nanowires to be single-crystalline and mostly oriented to the [111] direction [6].

\section{RESULTS AND DISCUSSION}

After the growth, samples were investigated under the optical microscope and scanning electron microscope (SEM). We observed variation in the properties of the nanowires according to the position on the sample. Mainly we observed three locations on the sample; area coated with gold (catalyzed region), the edge of the catalyzed region (contour), and finally the un-catalyzed region. Figure 1 shows the SEM image of the sample where all three regions are visible.

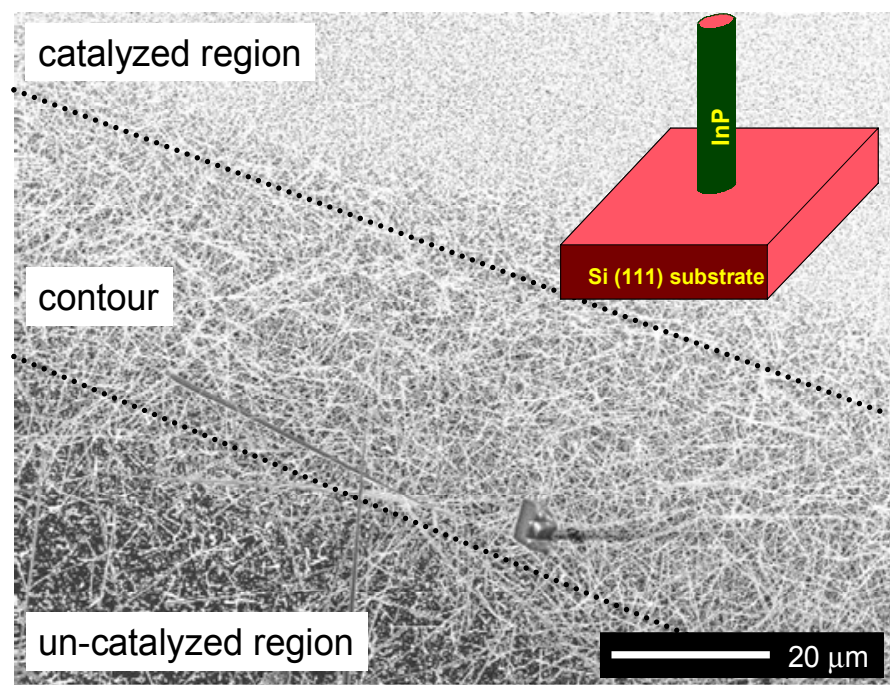

Figure 1. SEM image of the sample that shows all three distinct regions. The diagram depicts the growth method with the help of a metal catalysts $(\mathrm{Au})$. 
Nanowire growth on the catalyzed area was similar in both of the samples. The nanowires were 1 to $2 \mu \mathrm{m}$ long and we didn't observe a well defined preferred direction for the growth. Au catalyst particles at the tip of the nanowires were visible. The catalyst sizes were 23 to $56 \mathrm{~nm}$ in diameter for sample 1, and 50 to $90 \mathrm{~nm}$ for sample 2 . The difference in size of the $\mathrm{Au}$ particles is due to the initial thickness of the deposited Au layer. We observed that small Au particles (23 to $27 \mathrm{~nm}$ diameter) catalyzed cylindrical nanowires, while bigger particles caused either tapered nanowires with rectangular cross-section on sample 1 or irregular shaped and nanowires on sample 2. Rectangular cross-section InP nanowires grown in [001] direction on InP have been reported by Krishnamachari et.al. [7]. The sidewalls of these nanowires correspond to $<110>$ direction. Figure 2 shows the SEM image of nanowires grown on the Au catalyzed area.
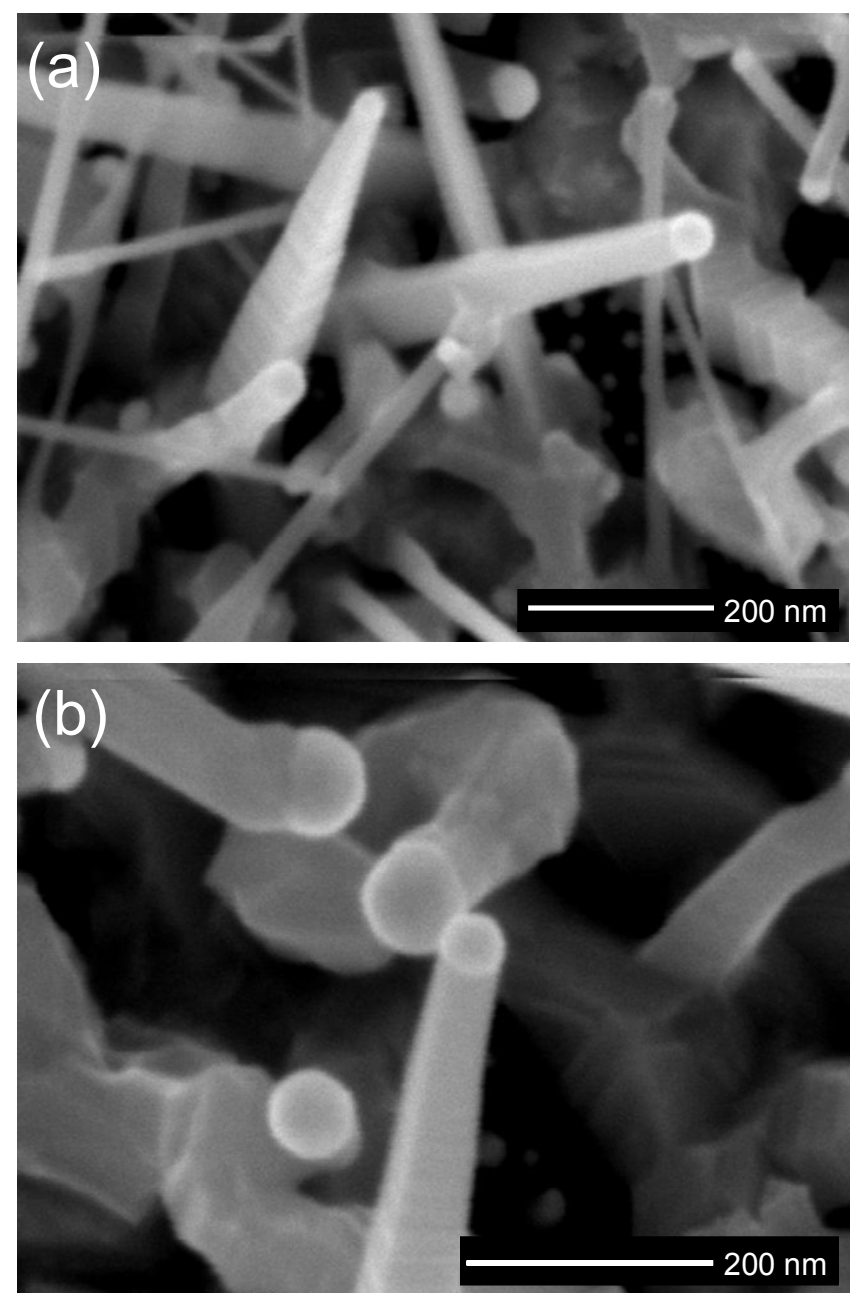

Figure 2. Nanowire growth on the catalyzed area of the sample 1 (a) and sample 2 (b). Although regular cylindrical nanowires were observed, most of the nanowires were irregular in shape.

At the edge of the catalyzed area, nanowires grew much longer than the nanowires at the catalyzed region as shown in Figure 3. We measured their length to be around 80 $\mu \mathrm{m}$ for sample 1 and around $60 \mu \mathrm{m}$ for sample 2. For both samples, the diameter of the nanowires is comparable to the size of the Au particles. The drastic increase in the growth rate can be explained with the migration of source atoms on the surface of the sample, which has been observed for III-V semiconducting nanowires like GaAs [8, 9] and GaP [10]. During the growth of the nanowire, the atoms were supplied to the catalyst either by (i) direct absorption of the source gas at the tip or (ii) the freely moving atoms which had decomposed on the un-catalyzed surface. At the edges of the gold deposited regions, $\mathrm{Au}$ catalyst particles were supplied with indium and phosphorus atoms by both methods. On the other hand, catalysts on the patterned area were closely placed and supplied with only direct absorption at the tip. For our growth conditions, the migration of atoms seems to play an important role in nanowire growth, which resulted in longer nanowires close to the un-catalyzed area. The density of long nanowires varies drastically between the samples. This variation can be explained with the size of the catalyst particles. The diameter of the nanowires is controlled by the size of the catalyst particles.
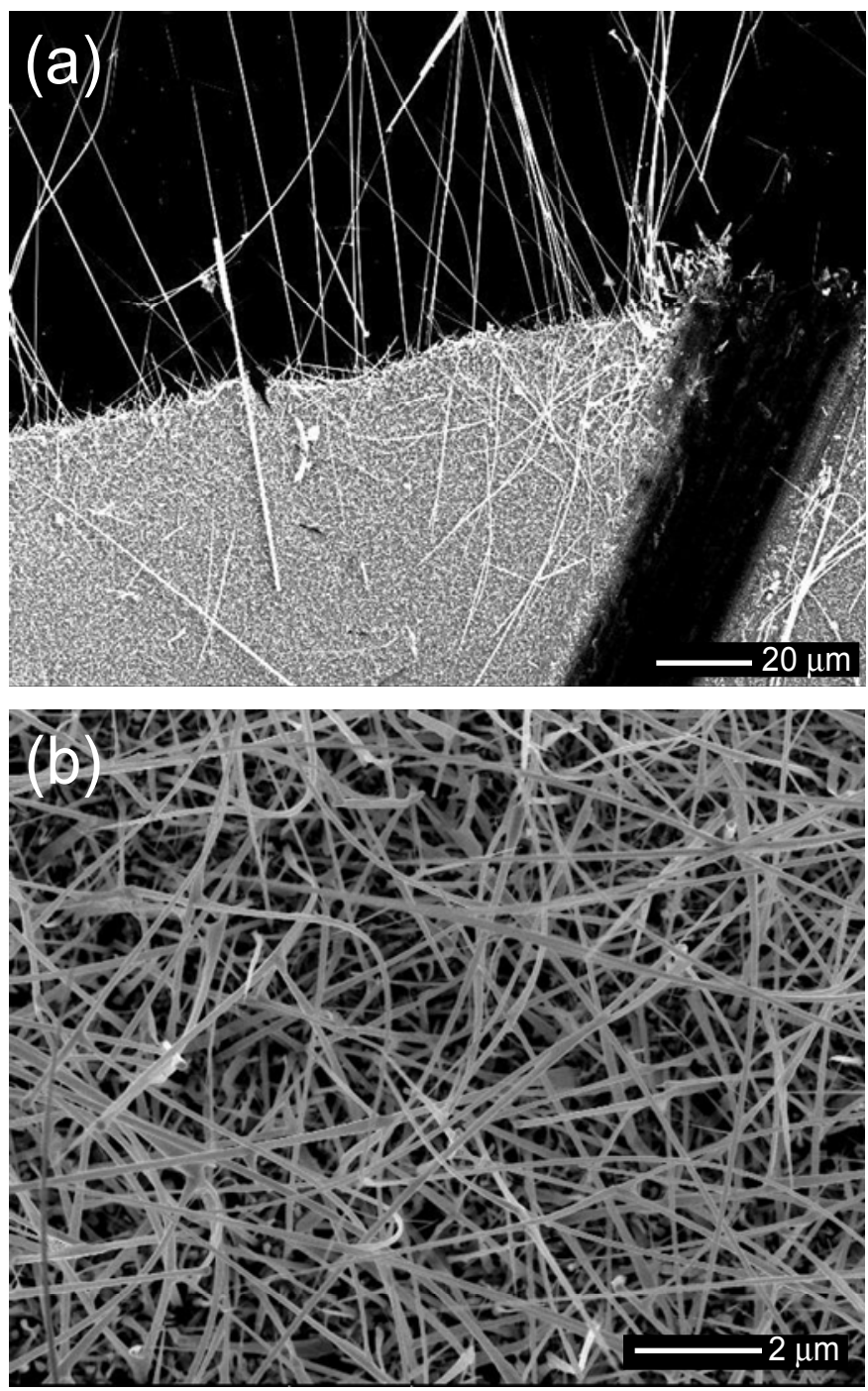

Figure 3. Nanowire growth at the edge of the catalyzed area of the sample 1 (a) and sample 2 (b). A great variation in the density of nanowires is seen. 
Unusually long nanowire growth was observed on the un-catalyzed area. We observed mainly two types of nanowires; (i) randomly oriented tapered nanonwires and (ii) regular nanowires. Multiple, tapered nanowires grew from the same root, and their growth directions were randomly oriented as seen on Figure 4. A possible cause of growth could be oxide-assisted growth or surface defects and ledges which initiated the growth of the nanowires. Nanowires with growth direction close to the normal of the sample, $<111>$ direction, were the longest among the nanowires on the same bunch. Their lengths were as long as $85 \mu \mathrm{m}$. As the growth direction departed from the normal, length of the nanowires decreased. These nanowires were 5 to $20 \mu \mathrm{m}$ in length. We could not observe any catalyst particle at the sharp tips of these nanowires.
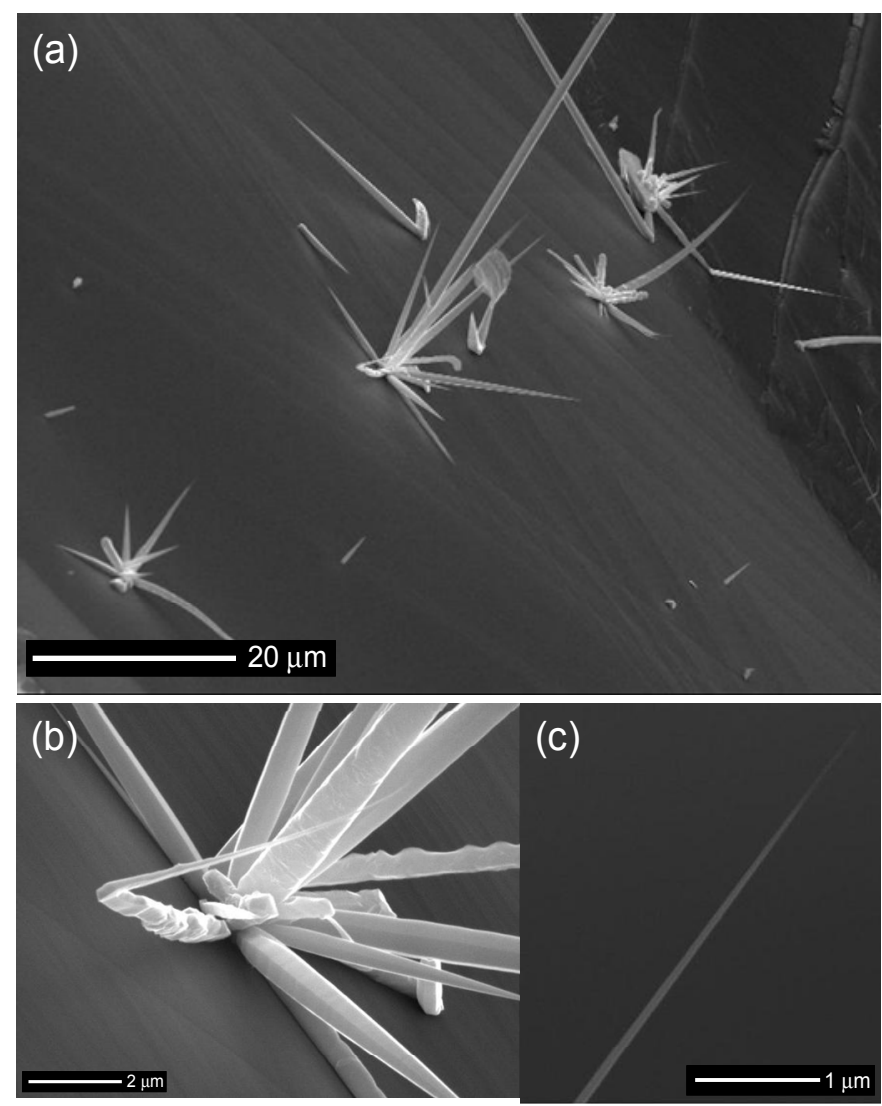

Figure 4. (a) Tapered hexagonal cross-section nanowires grown on the uncatalyzed area. Length of the short nanowires was 5 to $20 \mu \mathrm{m}$, while the long nanowires were up to $85 \mu \mathrm{m}$ long. (b) Details of the root of nanowires. (c) Sharp tip of the nanowires indicated that the nanowired grow without metal catalyst.

On the other hand, regular nanowires had grown quite far from each other normal to the surface of the sample as seen on Figure 5(a). They had almost the same diameter along the growth direction. Their diameters were 350 to $500 \mathrm{~nm}$ while their length ranged from 85 to $100 \mu \mathrm{m}$. We observed nanowires having aspect ratio of 250 . After careful observation of the tips of these nanowires, we found that these nanowires either had no catalyst or had a polycrystalline nanoparticle at the tip. The tips of nanowires can be seen in Figures 5(b) and 6(b). The nanoparticles had crystalline structure as seen in Figure 6(a). These nanoparticles might be InP nanocrystals formed during the growth or In-rich clusters. Due to low temperature growth, phase separation might have occurred to form In-rich clusters [11]. Both type of nanowires had hexagonal cross-section. Hexagonal cross-section nanowires have also been reported for catalyst-free growth of GaAs nanowires on GaAs substrates [9].

A recent theoretical study has suggested a critical value in the diameters of coherent and epitaxial nanowires when grown on highly lattice mismatched substrates [12]. Above this critical diameter, the growth is suggested to be unstable. Based on this theoretical work, the growth of InP nanowires on $\mathrm{Si}$ surfaces with $8 \%$ lattice mismatch is expected to generate a maximum diameter of $\sim 60 \mathrm{~nm}$. However, we observed nanowires with diameter as high as $500 \mathrm{~nm}$ in this experiment. More work is in progress to develop an understanding of this discrepancy.
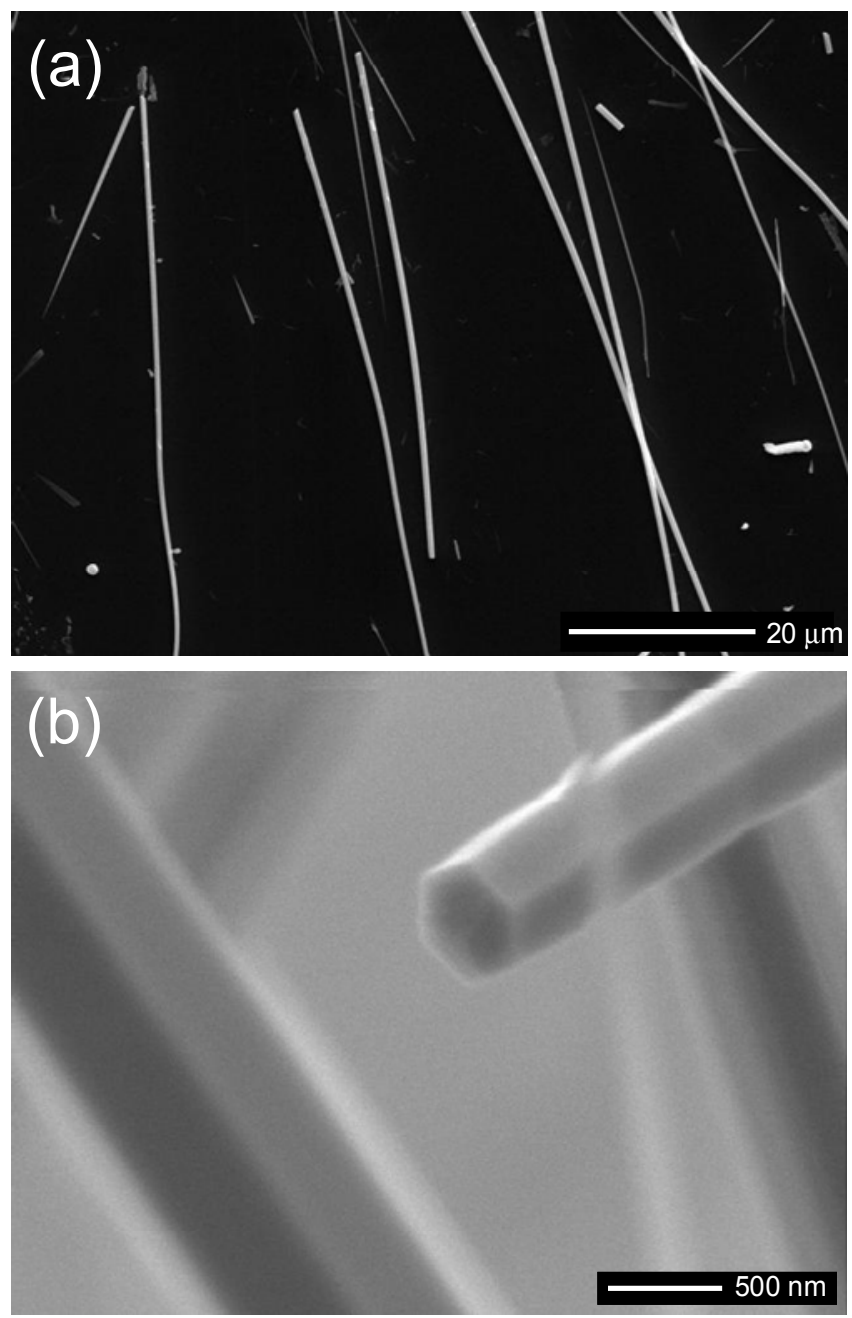

Figure 5. (a) Regular hexagonal cross-section nanowires grown on uncatalyzed area. Length of these nanowires was around $100 \mu \mathrm{m}$. (b) Tip of one of these nanowires. 

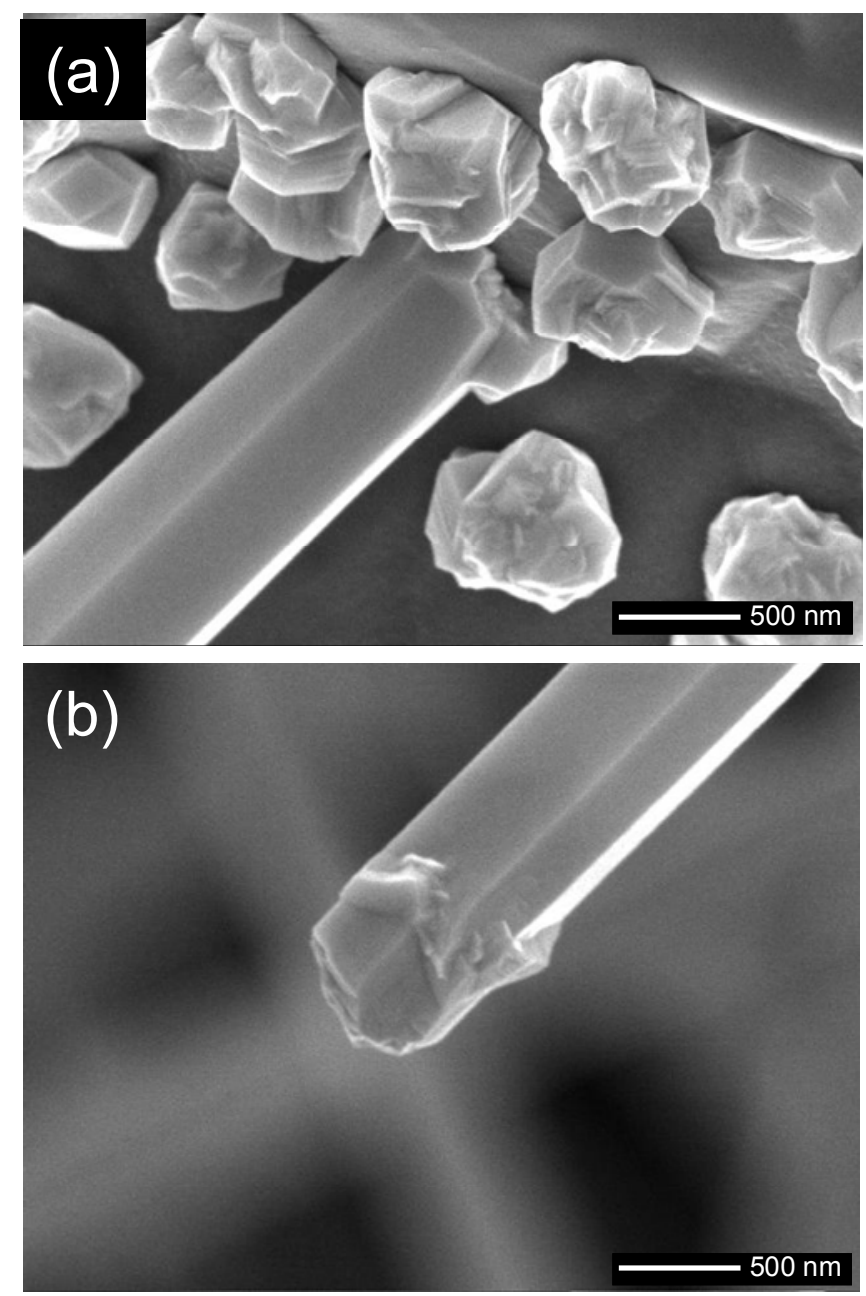

Figure 6. (a) Nanoparticle deposition on the surface of silicon. (b) Tip of one of the long nanowires show that these particles catalyze the growth.

\section{CONCLUSION}

We have observed the heteroepitaxial growth of InP nanowires on $\mathrm{Si}$ substrate with drastically different growth rates. We believe, the density and the size of catalyst particles determine the growth rate and the diameter of the nanowire. Very long self catalyzed InP nanowires showed that high aspect ratio nanowires can be grown on Si substrate. Detailed investigation is needed to control the diameter and the growth rate of self catalyzed nanowires. The $\mathrm{InP}$ nanowires can be integrated between Si electrodes using our nanowire bridging technique for further electrical and optical characterization [13, $14]$.

\section{REFERENCES}

[1] H. Mori, M. Tachikawa, M. Sugo, and Y. Itoh, "GaAs heteroepitaxy on an epitaxial Si surface with a low temperature process," Applied Physics Letters, vol. 63, pp. 1963-1965, 1993.

[2] A. Krost, F. Heinrichsdorff, D. Bimberg, and H. Cerva, "InP on Si(111): Accomodation of lattice mismatch and structural properties," Applied Physics Letters, vol. 64, pp. 769-771, 1994.

[3] M. T. Björk, B. J. Ohlsson, T. Sass, A. I. Persson, C. Thelander, M. H. Magnusson, K. Deppert, L. R. Wallenberg, and L. Samuelson, "Onedimensional steeplechase for electrons realized," Nano Letters, vol. 2, pp. 87-89, 2002.

[4] M. S. Gudiksen, L. C. Lauhon, J. Wang, D. C. Smith, and C. M. Lieber, "Growth of nanowire supperlattice structures for nanoscale photonics and electronics," Nature, vol. 415, pp. 617-620, 2002.

[5] Y. Watanabe, H. Hibino, S. Bhunia, K. Tateno, and T. Sekiguchi, "Sitecontrolled InP nanowires grown on patterned Si substrates," Physica E, vol. 24, pp. 133-137, 2004.

[6] S. S. Yi, G. Girolami, J. Amano, M. Saif Islam, T. I. Kamins, and S. Sharma, "InP nanobridges epitaxially formed between two vertical $\mathrm{Si}$ surfaces," presented at 5th IEEE Conference on Nanotechnology, Nagoya, Japan, 2005.

[7] U. Krishnamachari, M. Borgstorm, B. J. Ohlsson, N. Panev, L. Samuelson, W. Seifert, M. W. Larsson, and L. R. Wallenberg, "Defectfree InP nanowires grown in [001] direction on InP (001)," Applied Physics Letters, vol. 85, pp. 2077-2079, 2004.

[8] K. Haraguchi, K. Hiruma, K. Hosomi, M. Shirai, and T. Katsuyama, "Growth mechanism of planar-type GaAs nanowhiskers," Journal of Vacuum Science and Technology B, vol. 15, pp. 1685-1687, 1997.

[9] J. Noborisaka, J. Motohisa, and T. Fukui, "Catalyst-free growth of GaAs nanowires by selective-area metlorganic vapor-phase epitaxy," Applied Physics Letters, vol. 86, pp. 213102 1-3, 2005.

[10] K. A. Dick, K. Deppert, M. W. Larsson, T. Martensson, W. Seifert, L. R. Wallenberg, and L. Samuelson, "Synthesis of branched nanotrees by controlled seeding of multiple branching events," Nature Materials, vol. 3, pp. 380-384, 2004.

[11] J. Wu, J. Li, G. Cong, H. Wei, P. Zhang, W. Hu, X. Liu, Q. Zhu, Z. Wang, Q. Jia, and L. Guo, "Temperature dependence of the formation of nano-scale indium clusters in InAlGaN alloys on $\mathrm{Si}(111)$ substrates," Nanotechnology, vol. 17, pp. 1251-1254, 2006.

[12] E. Ertekin, P. A. Greaney, T. D. Sands, D.C. Chrzan, "Equilibrium analysis of Lattice-mismatched nanowire heterostructures", Mat Res. Soc. Symp. Proc. vol 737, pF10.4.1, 2003.

[13] M. Saif Islam, S. Sharma, T. I. Kamins, and R. Stanley Williams, "Ultrahigh-Density Semiconductor Nano-bridges Formed Between two Semiconductor Surfaces," Nanotechnology, vol. 15, pp. L5-L8, 2004

[14] M. Saif Islam , S. Sharma, T. I. Kamins, and R. Stanley Williams, "A novel interconnection technique for manufacturing nanowire devices," Applied Physics A, vol. 80, pp 1133-1140, 2005. 